\title{
Standard and non-standard neutrino physics at reactor experiments
}

\author{
David V. Forero*ab† \\ ${ }^{a}$ Universidad de Medellín, Medellín, Colombia \\ ${ }^{b}$ Instituto de Física Gleb Wataghin, UNICAMP, Campinas-SP, 13083-859, Brazil \\ E-mail: dvanegas@udem.edu.co
}

\begin{abstract}
In parallel to the standard oscillation physics program it is well known multi-detector reactor neutrino experiments are able to probe new physics scenarios, for instance, new oscillation phases driven by an extra light sterile neutrino and new neutrino interactions (NSI). Here we review and update the flavor non-universal and flavor universal constraints on the charged-current NSI parameters. We also study correlations between the NSI and the standard parameters.
\end{abstract}

The 20th International Workshop on Neutrinos (NuFact2018)

12-18 August 2018

Blacksburg, Virginia

\footnotetext{
* Speaker.

†I would like to thank the conveners of the 'Neutrinos beyond PMNS' session for accepting my contribution, and also my collaborators S. Agarwalla and M. Tórtola.
} 
Introduction: Neutrinos produced by fusion processes in the Sun, and as a byproduct of cosmic rays interacting with the Earth atmosphere, and also antineutrinos produced at reactor facilities, have been detected and studied for several decades. This wealth of observations is consistent with neutrino oscillations which has been established as the leading mechanism behind the observed neutrino flavor conversion. In the last few years, reactor and accelerator based experiments have played a major role towards the completion of the so called three active neutrino framework [1]. In particular, multi-detector reactor neutrino experiments of $\sim 1 \mathrm{~km}$ baseline have measured the reactor mixing angle with a precision $\sim 3 \%$. A non-zero reactor mixing angle has opened the possibility to measure the last missing parameter in this simplest picture, the Dirac CP phase encoding the possibility that leptons violate the charge-parity symmetry. Measuring the Dirac CP phase, establishing the neutrino mass ordering, and improving over the current precision on the atmospheric and solar parameters is the main aim of the 'standard' future neutrino program. Last but not least, and thanks to the required precision to fulfill the standard goals, one can also expect that New Physics (NP) might pop-up in the neutrino events. The origin of this NP can be for instance a new neutrino interaction, coupled to the neutrino sector, which at low energies 'manifests' itself as an effective four(or higher order) fermion operator(s). Due to the lack of a flavor structure and the unknown scale at which neutrino mass is generated (taking the neutrino mass models as a reference), it is then convenient to parametrize possible neutrino interactions (with fermions of the first family) in an effective way. These so called Non-Standard Interactions (NSI) are a modelindependent form to phenomenologically tackle the NP problem and have been widely studied in the literature. Matter effects at reactor neutrino experiments of $\sim 1 \mathrm{~km}$ baseline are practically negligible making them particularly sensitive to the so called Charged Current-like NSI which might be present at neutrino production/detection. Systematic errors are significantly reduced in modern reactor neutrino experiments thanks to the combination of near and far detectors, this in addition to their large statistics (of the order of a hundred thousand events) made them an interesting place to probe the mentioned NP scenario. In a previous work, a total-rate analysis to constrain the $\varepsilon_{e \alpha}^{u d}$ (with $\alpha=e, \mu, \tau$ ) NSI couplings was performed using the Daya Bay data released for 621-days of data taking. The focus of that work was on the robustness of the reactor mixing angle under the CC-like NSI, caring on the impact of the systematical errors. In this talk, an update of Ref. [2] was presented. Besides the use of the Daya Bay data released for 1230-days of data taking [4], also a spectral analysis is performed. This time, the focus is on the new physics phases encoded in the NSI couplings, also complementing the discussion on the interesting question of new sources of CP-violation.

Preliminary results: It is known that CC-like NSI, at the probability level, imply a redefinition of the reactor mixing angle whose approximate expression appear in Eqs. (2.18) and (2.19) in Ref. [2]. From this expression the number of extra NSI parameters is six, three complex couplings (one for each flavor). Regarding the statistical analysis, the main systematic error impacting our results was the total normalization error (mainly due to the error on the reactor flux total normalization) there labeled as norm. The main preliminary results were shown along slides 15 to 19 presented at the conference [3]. We opted for a one-parameter at time analysis, as shown in each slide. The corresponding 'shift' function that relates the measured reactor mixing angle in the presence of NSI was also shown. This last piece of information is useful to establish the freedom in the measured reactor mixing angle and also makes evident possible 'cancellation' of the main 
NSI contribution for particular values of the NSI and the Dirac CP phases. In cases where the NSI effects cancel out at first order in $|\varepsilon|$, the higher order corrections become the main contribution and weaker constraints are thus obtained. However, one can also include in the analysis a nonreactor bound on the reactor mixing angle as measured at Long-Baseline LBL experiments (shown in the vertical bands in the plots) which improves the constraints. Of course, this can be done by assuming the NP impacting neutrino oscillations at reactor experiments does not equally effect the LBL (accelerator based) experiments, or at least not at same level in the NSI expansion. A larger discussion on this topic will appear in Ref [5]. As an extra bonus, when marginalizing over the reactor mixing angle as given by the LBL-only measurement, interesting correlations in the $|\varepsilon|-\phi$ plane appear depicting not only the $\phi$-ranges where stronger NSI constraints can be obtained but also where the mentioned cancellations of the NSI contributions appear in the parameter space.

Main conclusion: Multidetector reactor neutrino experiments offer a clean probe of CC-like NSI. The $\theta_{13}$ determination is in general NOT robust under CC-like NSI (due to the effect of the phases) while the value of the NSI constraints is limited by our current knowledge of the 'absolute normalization of reactor neutrino fluxes'. This make us wonder if NP might be encoded in the systematic errors, which can be falsifiable with future experiments with a superb control of systematic errors.

\section{Acknowledgments}

D.V Forero was supported by the São Paulo Research Foundation (FAPESP) funding Grant No. 2014/19164-6 and 2017/01749-6, and also by BEPE Grant No. 2018/19365-2.

\section{References}

[1] P. F. de Salas, D. V. Forero, C. A. Ternes, M. Tortola and J. W. F. Valle, Phys. Lett. B 782, 633 (2018) doi:10.1016/j.physletb.2018.06.019 [arXiv:1708.01186 [hep-ph]].

[2] S. K. Agarwalla, P. Bagchi, D. V. Forero and M. Tortola, JHEP 1507, 060 (2015) doi:10.1007/JHEP07(2015)060 [arXiv:1412.1064 [hep-ph]].

[3] D.V. Forero, "Standard and non-standard neutrino physics at reactor experiments," https://indico.phys.vt.edu/event/34/contributions/662/.

[4] F. P. An et al. [Daya Bay Collaboration], Phys. Rev. D 95, no. 7, 072006 (2017) doi:10.1103/PhysRevD.95.072006 [arXiv:1610.04802 [hep-ex]].

[5] S. K. Agarwalla, D. V. Forero and M. Tortola, "Work in progress." 